\title{
Massive hemoptysis due to primary mucoepidermoid carcinoma of the lung in a 12-year-old
}

\author{
Authors: \\ Corresponding author: Lorraine du Toit-Prinsloo \\ Co-Authors: Belinda Kathleen Bunn
}

\begin{abstract}
Affiliation, degrees and addresses of the authors: (affiliation underlined, degrees in italic)
L du Toit-Prinsloo: $\quad$ Specialist / Lecturer; University of Pretoria; Department of Forensic Medicine, Private Bag X323, ARCADIA, 0007 SOUTH AFRICA MBChB, Dip For Med(SA)Path, FCForPath(SA), MMed(Path)(Forens)
\end{abstract}

BK Bunn:

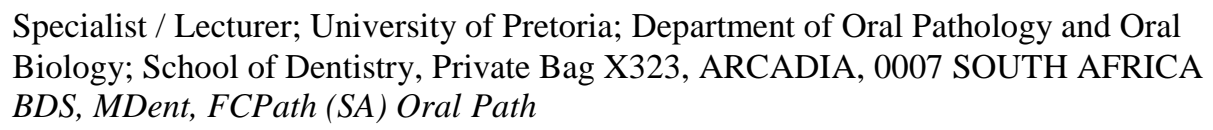

E-mail address, telephone and fax numbers of the corresponding author:

L du Toit-Prinsloo

Email: $\quad$ lorraine.dutoit@up.ac.za

Telephone numbers: $\quad+27(0) 123235298$ (work)

Fax number: $\quad+27(0) 123230921$

\section{Case Report}

A12-year-old African male patient presented with a history of progressive weight loss of several months duration and a complaint of persistent hemoptysis. Radiological examination (chest X-ray and computerized tomographic scan) confirmed the presence of a mass in the lower lobe of the right lung. Following hospitalization, the hemoptysis intensified and the patient was anesthetized for endobronchial examination and biopsy in order to establish the nature and extent of the pulmonary mass. Shortly after intubation, severe rapid intraparenchymal hemorrhage of the right lung occurred which led to sudden cardiac arrest. The patient was successfully resuscitated and was then admitted to the intensive care unit whilst still intubated. The endobronchosopic examination and biopsy procedure were abandoned and the right lung mass remained undiagnosed. A brain CT-scan performed shortly thereafter confirmed that hypoxic damage was present. The patient showed no improvement and finally succumbed one month later.

The case was referred for medico-legal post-mortem examination in accordance with South African legislation as a so-called "procedure associated/related death" as per the provisions of section 56 of the Health Professions Act 56 of 1974 (as amended by the Health Professions Amendment Act 29 of 2007) and the Inquests Act 58 of 1959.

The post mortem examination showed the deceased to be of average physique with a height of 1,4 meters, although he was cachectic and weighed only 29 kilograms (Body Mass Index =14,8). External examination showed a superficial pressure sore in the occipital region of the scalp. The brain was edematous with widening and flattening of the gyri and narrowing of the sulci. The brain weighed 1419 grams. On section, the cut 
surface appeared dusky pink in color. Diffuse hypoxic / ischemic change was noted on histological examination of representative tissue sections.

The right lung was enlarged and weighed 567 grams. A large, fairly well circumscribed lobulated tumor with a firm, rubbery texture on sectioning was identified within the lower lobe of the right lung. Cystic areas filled with yellow, gelatinous material was identified macroscopically (Figure 1). Furthermore, tumor infiltration of the adjacent pulmonary artery was also noted.

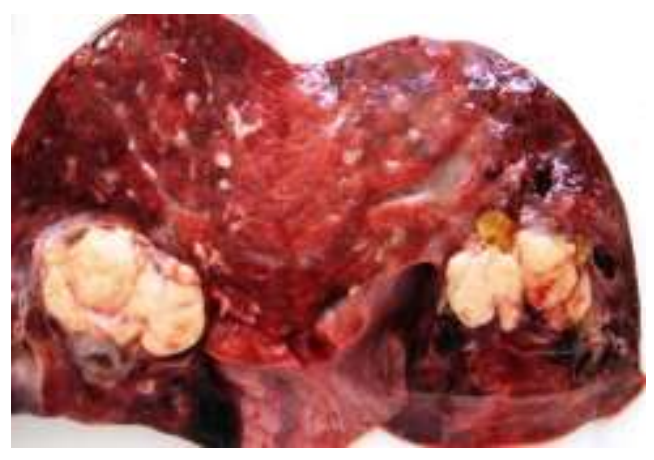

Figure 1: Macroscopic appearance of the tumour. A fairly well circumscribed lobulated tumour with a firm rubbery consistency with intervening cystic areas containing yellow mucoid material.

Histological examination of tissue sections from the lung mass shows an exophytic endobronchial and pulmonary infiltrate with a prominent intracystic component with intervening areas of solid tumor growth. Within the solid areas are epidermoid (squamoid) cells which are polygonal in shape with abundant eosinophilic cytoplasm and occasional intercellular bridges. There is no histological evidence of keratinization. There are admixed intermediate cells which are somewhat smaller in size and have uniform hyperchromatic nuclei. Occasional mucous producing cells with columnar and goblet cell appearances are identified scattered throughout as well as within the lining of the micro-cystic and larger cystic spaces (Figures $2 \mathrm{a}$ and $2 \mathrm{~b}$ ). The cystic spaces are filled with mucinous secretions. Histochemical stains (Periodic acid-Schiff and mucicarmine) confirm the presence of intracytoplasmic mucin production (Figure 3).

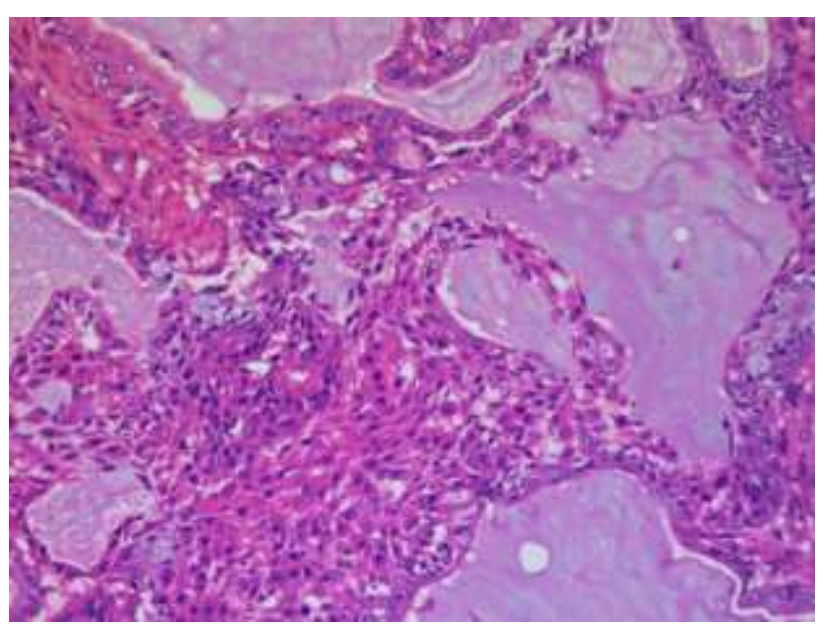

Figure 2a: Haematoxylin and eosin stained section, x200. Mucoepidermoid carcinoma of the lung comprising epidermoid, intermediate and mucous cells with both solid and intracystic components. 


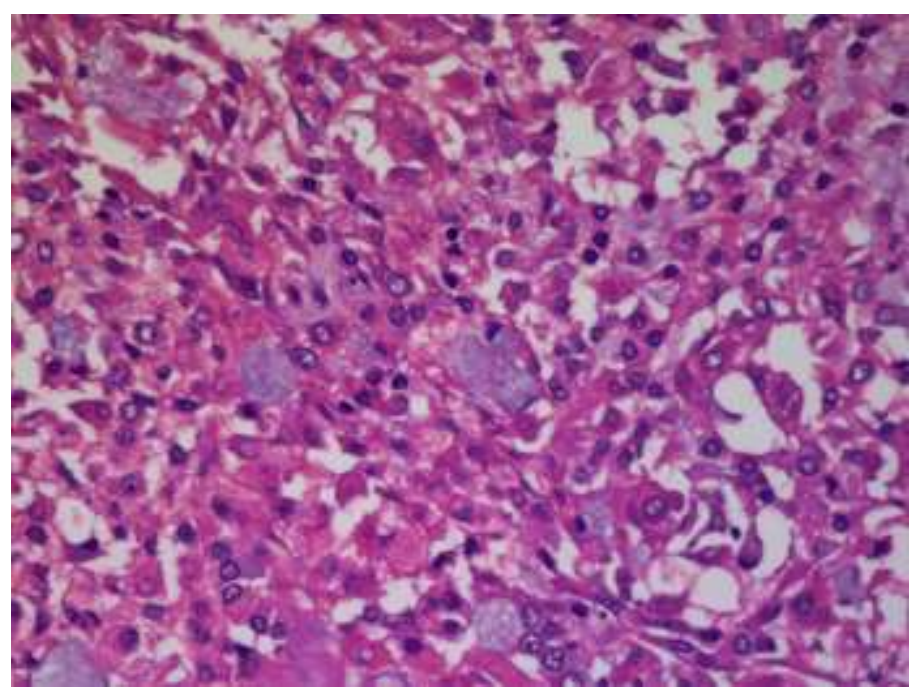

Figure 2b: Haematoxylin and eosin stained section, $x 400$. An area of solid tumour growth in which scattered mucous cells are discernable. These cells are surrounded by an admixture of squamous and intermediate cells.

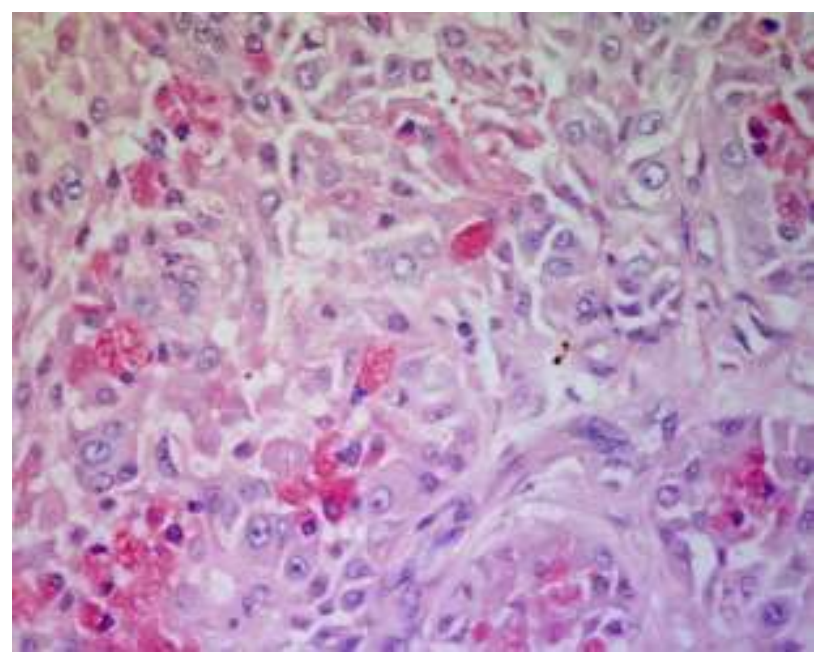

Figure 3: Mucicarmine special stain, $x 400$. Intracytoplasmic mucin production is highlighted within the mucous cells.

The tumor cell nuclei are largely vesicular with occasional areas of mild pleomorphism. The mitotic index is fairly low and there is no evidence of tumor-associated necrosis. Immunohistochemical staining for thyroid transcription factor-1 (TTF-1) as well as CK7 and CK20 were performed. CK7 was strongly and diffusely positive within the tumor cells (Figure 4). Immunostaining for CK20 and TTF-1 were both negative. 


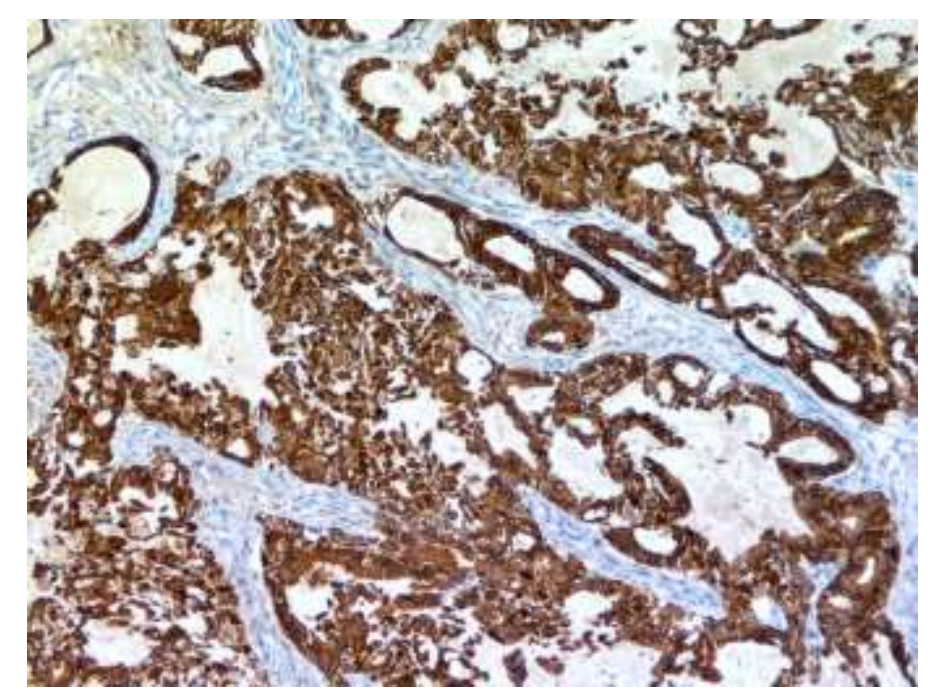

Figure 4: CK7 immunohistochemical stain x100. The tumour cells are strongly and diffusely positive for CK7. The staining highlights the cystic and solid areas of tumour cell growth.

The morphological, histochemical and immunophenotype of this neoplasm is in keeping with the presence of a mucoepidermoid carcinoma. The neoplasm is largely low-grade although it is seen to infiltrate the adjacent lung parenchyma quite extensively.

\section{Discussion}

Primary lung carcinomas are rare in children and frequently have non-specific clinical presentations including dyspnea, cough, wheezing and hemoptysis. The clinical features often mimic chronic obstructive pulmonary disease or infectious processes.[1,2] Primary salivary-type adenocarcinomas of the lung are an exceptionally rare subgroup of pulmonary neoplasms constituting less than one per cent of all primary neoplasms at this site.[3,4] Primary pulmonary neoplasms seldom occur in the pediatric population and are more usually metastatic.[5] In the South African review of primary pulmonary tumors in children admitted to the Red Cross War Memorial Children's Hospital in Cape Town between 1960 and 1991, a ratio between primary pulmonary tumors, secondary neoplasms and non-neoplastic lesions was determined to be 1:5:60.[6] Complicating the clinical presentation and accurate diagnosis of primary pulmonary neoplasms are their non-specific clinical features which typically mimic a range of non-neoplastic obstructive disease.[1,2] Most primary pulmonary neoplasms are malignant and their associated symptoms usually include cough, pneumonitis, fever, respiratory distress and hemoptysis.[1]

Salivary-type adenocarcinomas of the lung are a rare subgroup of primary pulmonary neoplasm which account for less than one per cent of all primary pulmonary malignancies.[3,4] These tumors are morphologically indistinct from their salivary gland analogues and arise within the submucosal glands of the tracheobronchial tree.[3,4] Mucoepidermoid carcinoma of the lung was first documented by Smetana and Liebow in 1952 and has been reported over a wide age range spanning from 3 to 78 -years. There is a slight male predilection and more than half of these patients will present before the age of 30-years.[1,2] Smoking or occupational lung 
exposures are not recognized risk factors for tumor development.[1,2] Within the pediatric population, tumors in older children over the age of 8-years are usually malignant. [7]

Clinically, mucoepidermoid carcinoma presents as an exophytic endobronchial mass which results in obstructive symptoms often followed by distal collapse / atelectasis of the lung parenchyma. Patients often complain of dyspnea and asthma-like features. The misdiagnosis of an infectious process such as pneumonitis or tuberculosis is frequently made. Secondary bronchi and the peripheral pulmonary parenchyma may also be involved by tumor but much less frequently. [2, 4]

The importance of appropriate radiological imaging, endobronchosopic examination and a high level of clinical suspicion are essential to avoid the misdiagnosis and inappropriate clinical management of these rare neoplasms at a pulmonary site. Most lung mucoepidermoid carcinomas are low-grade and have a good long-term prognosis and survival if resected early on. $[1,2]$

Mucoepidermoid carcinoma represents an adenocarcinoma composed of varying numbers of three cell types including mucous producing cells, intermediate (germinative) cells and epidermoid (squamoid) cells. [8] Mucoepidermoid carcinoma (MEC) of salivary glands represents the most common primary salivary gland malignancy in both the pediatric and adult populations with tumors arising predominantly within the paired major salivary glands and less frequently within the minor intra-oral glands.[8] Morphologically similar lesions have been described in the cervix, skin, breast and lung although data regarding the nature and prognosis are scanty due to the small series of cases reported at these sites. These neoplasms arise from the homologous exocrine glands located at these sites and recapitulate the histomorphology of almost the entire spectrum of neoplasms described in the salivary glands. $[1,3]$

The histological grading of primary salivary MEC has been exhaustively reviewed and is thus well established.[8,9] Primary pulmonary MEC is graded more simply into low-grade and high-grade neoplasms on the basis of cellular composition and cytological features.[10] Low-grade neoplasms tend to arise centrally within the tracheobronchial tree and seldom metastasize. They show a conspicuous cystic component and predominance of mucous producing cells and bland vesicular nuclei.[11] Radiologically, low-grade MEC of the lung is fairly well-circumscribed and of homogenous density.[12] High-grade MEC of the lung usually arises more peripherally and are histologically characterised by more diffuse solid tumor growth with a greater abundance of both intermediate and epidermoid cells. The intracystic component is less conspicuous. [3, 11] The epidermoid cells do not show features of keratinization although intercellular bridges may be noted. The tumor cells have increased nuclear hyperchromatism and pleomorphism with an increased mitotic rate of usually more than four mitoses in ten high power fields. [3] There are frequently areas of tumor associated necrosis. Radiologically, these neoplasms are more ill-defined and of heterogeneous density. [12]

The histological spectrum of salivary-type adenocarcinomas of the lung has increased and therefore the accurate diagnosis thereof relies on an increased awareness and familiarity of these neoplasms occurring at this unusual site. Bronchoscopic washings or fine needle aspirate cytology may not always be definitive due to the inherent 
microscopic heterogeneity of this subgroup of neoplasms with many tumors having a prominent stromal component. Immunohistochemical staining and molecular testing may allow for easier distinction. MEC is positive for CK5/6, CK7, p63 and p40 whilst being negative for CK20, TTF-1 and naspin-A.[8,10,13-14] This is also useful in facilitating the distinction between primary pulmonary epithelial neoplasms and the subgroup of salivary-type adenocarcinomas.[8,10] Histochemical stains to confirm the presence of intracytoplasmic mucin production by the mucous cell component is essential in the diagnostic process as even the high-grade neoplasms invariably have scattered mucous cells even if the lower-grade areas are only focal.[3,15] Furthermore, biopsy representivity is thus important for all of these features to be recognized.

Head and neck MEC have recently been shown to have a recurrent translocation $(t 11 ; 19)$ which involves Mucoepidermoid carcinoma translocated-1 on chromosome 19p13 (MECT1) and Mastermind-like 2 on chromosome 11q21 (MAML2).[13,14] Fusion positivity is strongly correlated with lower histological grade in these neoplasms. A recently documented series of 26 cases of primary pulmonary MEC were shown to be fusion positive by means of fluorescent in-situ hybridization in 12 of the 18 primary pulmonary MEC cases. [14]

The possibility of metastatic salivary disease should always be ruled out in pulmonary neoplasms in this tumor subgroup and should be distinguished from primary pulmonary adenosquamous carcinoma as well as squamous cell carcinoma at this site. Adenosquamous carcinoma usually has a distinct tumor cell interface between an adenocarcinoma component and squamous cell carcinoma with intermingling of these neoplasms with diffuse high-grade cytological features. Primary pulmonary MEC by contrast will almost always have areas of lowgrade cytology at least focally.[3] High-grade primary pulmonary MEC may closely resemble squamous cell carcinoma although tumors show a distinct lack of keratinization and are not associated with an in-situ / dysplastic component within the overlying epithelium.[3]

Clinical staging is used together with histological tumor grade and tumor location and resectability in order to determine the lesional prognosis. [1,9] Complete surgical resection remains the mainstay of treatment whilst the exact role of chemoradiation is largely unknown. Indications for adjunctive therapy are an irresectable tumor or positive surgical margins. Therapeutic outcome and success is not well understood due to the small series of these neoplasms at a pulmonary site of origin.[12] The identification of the recurrent $t(11: 19)$ within a large number of MEC has spurred on research into the development of novel therapeutic targets in the treatment of these neoplasms.[11,14]

\section{References:}

1. Pandey D, Garg PK, Jakhetiya A, Pandey R, Bhoriwal S, Nath D et al. Surgical experience of primary salivary gland tumors of lung: A case series. (Short title: Primary salivary gland tumours of the lung) Int J Surg. 2015; 21: 92-6.

2. Belgod SR, Reddy RHV,Kumar SP. Mucoepidermoid carcinoma of the lung: a rare entity. Oxf Med Case Reports. 2015;2: 203-5. 
3. Falk N, Weissferdt A, Kalhor N, Moran CA. Primary Pulmonary Salivary Gland-type Tumors: A Review and Update. Adv Anat Pathol. 2016; 23(1):13-23.

4. Molina JR, Aubry MC, Lewis JE, Wampfler JA, Williams BA, Midthun D et al. Primary salivary gland-type lung cancer. Spectrum of clinical presentation, histopathological and prognostic factors. Cancer 2007;110(10):2253-9

5. Welsh JH, Maxson T, Jaksic T, Shahab I, Hicks J. Tracheobronchial mucoepidermoid carcinoma in childhood and adolescence: case report and review of the literature. Int J Pediatr Otolaryngol. 1998; 45: 265-73.

6. Cohen MC, Kaschula RO. Primary pulmonary tumors in childhood: a review of 31 years' experience and the literature. Pediatr Pulmonol. 1992; 14(4): 222-32.

7. Roby BB, Drehner D, Sidman JD. Pediatric tracheal and endobronchial tumors: an institutional experience. Arch Otolaryngol Head Neck Surg. 2011; 137(9): 925-9.

8. goode RK, El-Naggar. Mucoepidermoid carcinoma in: Barnes L, Eveson JW, Reichart P, Sidranksy D. World Health Organisation Classification of Tumours. Pathology \& Genetics - Head and Neck Tumours. IARC Press Lyon, 2005. p219-220

9. Brandwein MS, Ivanov K, Wallace DI, Hille JJ, Wang B, Fahmy A et al. Mucoepidermoid carcinoma: a clinicopathologic study of 80 patients with special reference to histological grading. Am J Surg Pathol. 2001; 25(7): 835-45.

10. Wick MR, Marx A, Muller-Hermelink HK, Strobel Ph. Mucoepidermoid carcinoma in: Travis WD BE, Muller-Hermelink HK, Harris CC. World Health Organisation Classification of Tumours. Pathology \& Genetics. Tumours of the lung, pleura, thymus and heart. IARC Press, Lyon 2004, p. 176.

11. Thomas D, Modi Y, Dorai B, Guron G. A rare case of lung carcinoma with mucoepidermoid histopathology: a case report and review of the literature. Ann Clin Lab Sci. 2015; 45(2): 219-21.

12. Wang YQ, Mo YX, Li S, Luo RZ, Mao SY, Shen JX. Low-Grade and High-Grade Mucoepidermoid Carcinoma of the Lung: CT Findings and Clinical Features of 17 Cases. AJR Am J Roentgenol. 2015; 205:1160-6.

13. Roden AC, Garcia JJ, Wehrs RN, Colby TV, Khoor A, Leslie KO, et al. Histopathological, immunophenotypic and cytogenic features of pulmonary mucoepidermoid carcinoma. Mod Pathol 2014;27:1479-88

14. Huo Z, Wu H, Li J, Li S, Wu S, Liu Y, et al. Primary pulmonary mucoepidermoid carcinoma: Histopathological and moleculargenetic studies of 26 cases. PLosOne 2015;10(11):e0143169

15. Abu Saleh WK, Aljabbari O, Ramchandani M. Mucoepidermoid Carcinoma of the Tracheobronchial Tree. Methodist Debakey Cardiovasc. 2015; 11(3): 192-4. 